\title{
Synthese des 19-Oxo-3-aza-A-homo-5 $\beta$-androstans
}

\author{
Gerhard Habermehl und Arthur Haaf
}

Institut für Organische Chemie der Technischen Hochschule Darmstadt

\author{
(Z. Naturforsch. 25 b, 191-195 [1970] ; eingegangen am 15. September 1969)
}

\begin{abstract}
Zur Untersuchung der wesentlichen Reaktionsschritte der Synthese des Cycloneosamandions wurde als Modellsubstanz das 19-Oxo-3-aza-A-homo-5 $\beta$-androstan, ausgehend vom $3 \beta$-Hydroxy-5achlor-6 $\beta$,19-epoxy-androstan-17-on dargestellt. Die spektroskopischen Daten stehen im Einklang mit der erwarteten Struktur.
\end{abstract}

Vor kurzem berichteten wir über die Synthese des Cycloneosamandions ${ }^{1}$. Als Modellsubstanz hatten wir zuvor die Darstellung des 19-0xo-3-aza-A-homo$5 \beta$-androstans (12 a bzw. b) durchgeführt, die wir im folgenden beschreiben wollen.

19-Oxo-3-aza-A-homo-5 $\beta$-androstan (12 b) unterscheidet sich vom Cycloneosamandion lediglich durch das Fehlen der Sauerstoffunktion an C-16.

Die Ketogruppe an C-17 des als Ausgangsmaterial verwendeten 3 -Hydroxy-5 $\alpha$-chlor-6 $\beta$, 19-epoxy-androstan-17-ons $(\mathbf{1})^{2}$ haben wir nach der Methode von BARTON et al. ${ }^{3}$ über das Hydrazon (2) und anschließender Umsetzung mit Jod entfernt; dabei erhielten wir das $3 \beta$-Hydroxy-5 $\alpha$-chlor-17-jod- $6 \beta$, 19 epoxy- $\Delta^{16}$-androsten ( 3). $\mathrm{LiAlH}_{4}$-Reduktion von 3 führte zu $3 \beta$-Hydroxy-5 $\alpha$-chlor- $6 \beta$,19-epoxy- $\Delta^{16}$. androsten (4), das noch mit nicht umgesetztem 3 verunreinigt war. Dieses Gemisch ließ sich nur schwer präparativ trennen; wir haben daher auf eine Reinigung verzichtet und das Rohprodukt direkt mit Chromsäure in Aceton oxydiert; dabei erhielten wir mit $5 \alpha$-Chlor-17-jod-6 $\beta$,19-epoxy- $\Delta^{16}$-androsten-3-on verunreinigtes $5 \alpha$-Chlor-6 $\beta, 19$-epoxy- $\Delta^{16}$-androsten3 -on $(\mathbf{5} \mathbf{a})$. Wir haben dieses Gemisch wiederum direkt zur Dehydrohalogenierung mit Kaliumacetat in Methanol eingesetzt. Nach dieser Reaktion konnten die beiden Komponenten durch PSC an $\mathrm{SiO}_{2}$ getrennt werden und wir erhielten so das $6 \beta, 19-\mathrm{Ep}-$ oxy- $\Delta^{4.16}$-androstadien-3-on $(6$ a) und das 17-Jod$6 \beta, 19$-epoxy- $\Delta^{4.16}$-androstadien-3-on $(6 \mathrm{~b})$ als farblose Kristallisate.

Die Spaltung der 6 $\beta, 19$ - Ätherbrücke erfolgte mit $\mathrm{Zn} /$ Eisessig ${ }^{2}$ und lieferte 19-Hydroxy- $\Delta^{4.16}$-androstadien-3-on ( 7 a) bzw. 19-Hydroxy-17-jod- $\Delta^{4.16}$.

1 G. Habermehl u. A. HaAf, Z. Naturforsch. 23 b, 1551 [1968].

2 H. Ueberwasser, K. Heusler, J. Kalvoda, Ch. Meystre, P. Wieland, G. Anner u. A. Wettstein, Helv. chim. Acta 46, 344 [1963].

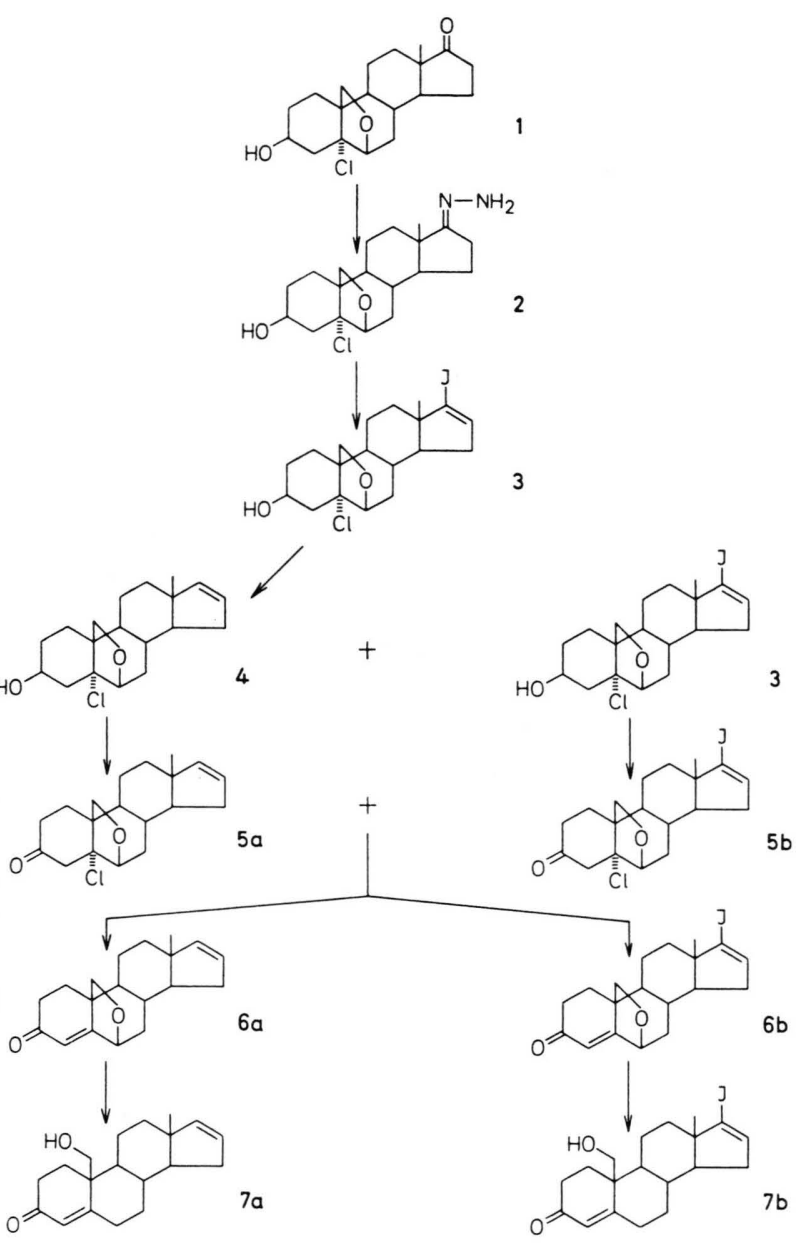

androstadien-3-on (7 b) . Durch Hydrierung mit Pd-C (10-proz.) im alkalischen Medium ${ }^{4}$ konnte sowohl aus $7 \mathbf{a}$, wie auch aus $7 \mathbf{b}$ das $19-$ Hydroxy- $5 \beta$-andro-

3 D. H. R. Barton, R. E. O'Brien u. S. Sternhell, J. chem. Soc. [London] 1962, 470.

4 J. B. Jones u. P. Price, Canad. J. chem. 44, 999 [1966]. 


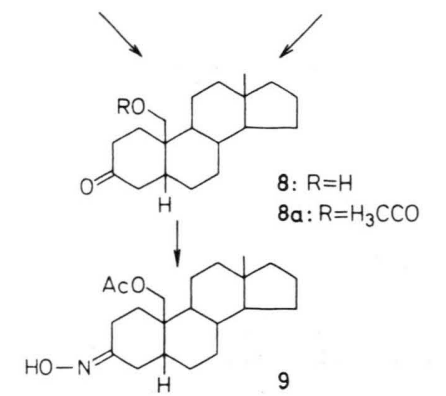

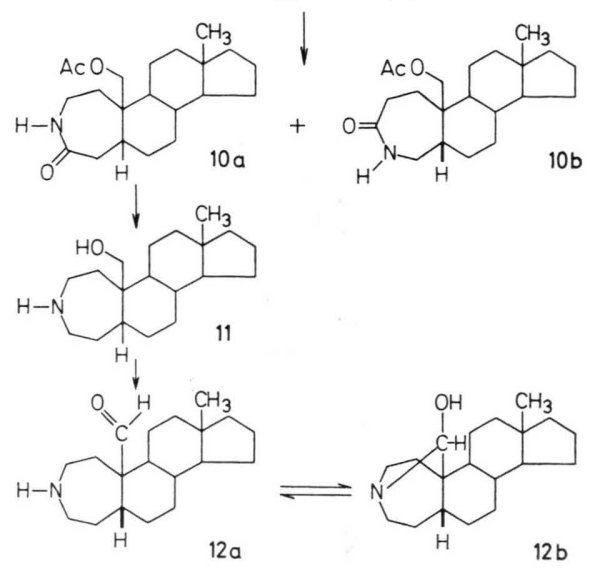

stan-3-on (8) gewonnen werden. Die $\beta$-Konfiguration des Wasserstoffs an C-5 geht eindeutig aus dem negativen C ot to n-Effekt der CD-Kurve der 19-Acetylverbindung $(8 \mathrm{a})$ hervor. $\left(\lambda_{\max }=295 \mathrm{~m} \mu\right.$; $\Delta \varepsilon=0,633$.)

Vor Einführung des Stickstoffs in den Ring A des Steroidskeletts wurde zunächst die 19-Hydroxygruppe von 8 acetyliert. Das Oxim des so erhaltenen 19-Acetoxy-5 $\beta$-androstan-3-ons (9) lagerte sich mit Thionylchlorid bei $-20{ }^{\circ} \mathrm{C}^{6}$ nach B e c k m a n n um. Die beiden isomeren $\varepsilon$-Laktame 10 a und 10 b konnten durch präp. Schichtchromatographie an $\mathrm{Al}_{2} \mathrm{O}_{3}$ (Merck) Typ $\mathrm{T}^{5}$ getrennt werden.

Die Stellung des Stickstoffs im Ring A des Steroidskeletts wurde aus dem NMR-Spektrum durch Vergleich mit $\varepsilon$-Laktamen bekannter Struktur ${ }^{5,7}$ bestimmt.

So liefert das Kernresonanzspektrum von 19. Acetoxy-3-aza-4-oxo-A-homo-5 $\beta$-androstan (10 a) (Abb. 1) neben dem Signal für die C-18-Methylgruppe bei $\tau=9,29(3 \mathrm{H})$ und dem Signal für die Methylgruppe der 19-Acetoxygruppe bei $\tau=7,94$

5 G. Habermehl u. A. Haaf, Liebigs Ann. Chem. 722, 155 [1969].

6 C. W. Shoppee u. G. Krueger, J. chem. Soc. [London] 1962,470
$(3 \mathrm{H})$ das bei allen 3-Aza-4-oxo-A-homo-steroiden auftretende Multiplett zentriert um $\tau=6,88(2 \mathrm{H})$, das wir den Protonen an C-2 zuordnen. Die Methylenprotonen der $-\mathrm{CH}_{2}-\mathrm{O}-\mathrm{CO}-\mathrm{CH}_{3}$-Gruppierung an C-10 treten als Dublett bei $\tau=5,55(\mathrm{~J}=$ $11 \mathrm{~Hz})$ und $\tau=6,06(J=11 \mathrm{~Hz})$ auf.

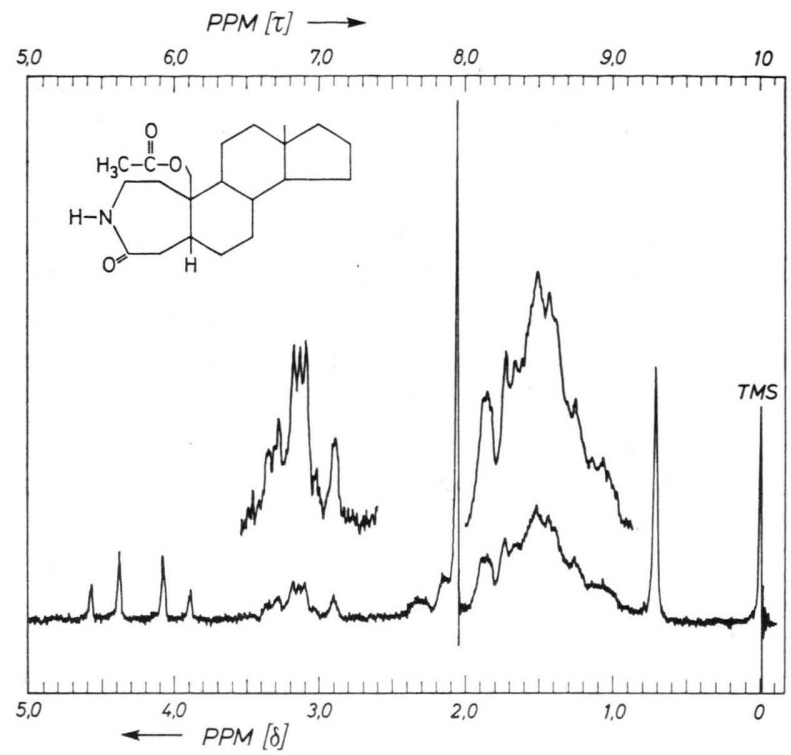

Abb. 1. $60 \mathrm{MHz}-K e r n r e s o n a n z s p e k t r u m$ von 19-Acetoxy-3-aza4-oxo-A-homo-5 $\beta$-androstan (10 a) in $\mathrm{CDCl}_{3}$; TMS als interner Standard.

Im NMR-Spektrum von 19-Acetoxy-4-aza-3-oxoA-homo-5 $\beta$-androstan ( 10 b) (Abb. 2) treten außer dem Multiplett bei $\tau=6,88$, die oben genannten Signale an denselben Stellen auf. Statt dieses Multipletts findet man eine Signalgruppierung von $\tau=7,0$ bis $\tau=7,7$ und von $\tau=6,0$ bis $\tau=6,5$. Letztere ist teilweise von den vier Linien des AB-Systems der Methylenprotonen der $-\mathrm{CH}_{2}-\mathrm{O}-\mathrm{CO}-\mathrm{CH}_{3}$ Gruppe an C-10 verdeckt. Wir ordnen die Signale bei $\tau=7,0$ bis $\tau=7,7$ und $\tau=6,0$ und $\tau=6,5$ den Protonen an C-4 a zu; auch hier entspricht das Kernresonanzspektrum von $\mathbf{1 0} \mathrm{b}$ dem anderer 4-Aza-3oxo-A-homo-Steroide.

Durch Reduktion von $\mathbf{1 0}$ a mit $\mathrm{LiAlH}_{4}$ in siedendem THF erhielten wir das Amin 11, welches als Hydrojodid charakterisiert wurde (IR-Spektrum Abb. 3). Oxydation von 11 mit $\mathrm{CrO}_{3}$ in 3-proz. $\mathrm{H}_{2} \mathrm{SO}_{4}{ }^{8}$ lieferte das 19-0xo-3-aza-A-homo-5 $\beta$-andro-

7 G. Habermehl u. A. Haaf, Liebigs Ann. Chem. 723, 181 [1969]. 


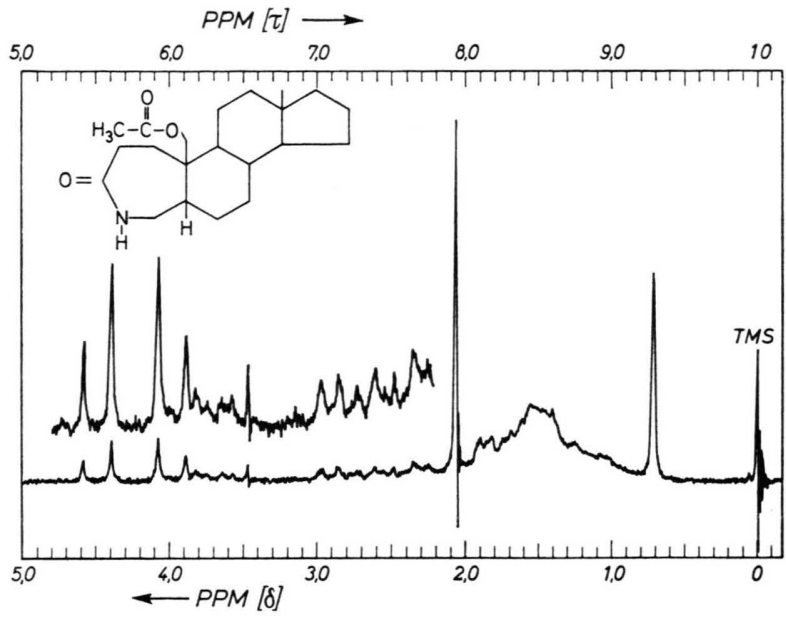

Abb. 2. $60 \mathrm{MHz}-\mathrm{Kernresonanzspektrum} \mathrm{von} \mathrm{19-Acetoxy-4-aza-}$ 3-oxo-A-homo-5 $\beta$-androstan $(\mathbf{1 0} \mathbf{b})$ in $\mathrm{CDCl}_{3}$; TMS als interner Standard.

stan (12 a). Diese Verbindung kann jedoch nicht als solche gefaßt werden, sie bildet sofort die entsprechende Cycloverbindung 12 b mit der Carbinolamingruppierung.

Im IR-Spektrum des Hydrojodids von $\mathbf{1 2} \mathbf{b}$ (Abb. 4) findet sich dementsprechend keine $\mathrm{C}=\mathrm{O}$ Bande für die freie Aldehydgruppe bei $1720 \mathrm{~cm}^{-1}$.

\section{Beschreibung der Versuche}

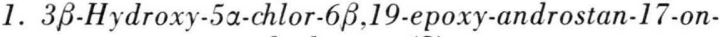 hydrazon (2)}

57,4 g $3 \beta$-Hydroxy-5 $\alpha$-chlor-6 $\beta, 19$-epoxy-androstan17-on (1) (dargestellt nach l. c. ${ }^{2}$ ) wurden in $500 \mathrm{ml}$ heißem Äthanol gelöst und $58 \mathrm{ml}$ Hydrazinhydrat (80-proz.) zugegeben. Das Reaktionsgemisch wurde über Nacht bei Raumtemperatur stehengelassen, mit etwas Wasser verdünnt und das Lösungsmittel soweit i. Vak. abgezogen, bis die Kristallisation begann. Nach Eiskühlung wurde der kristalline Niederschlag abgesaugt. Man erhielt 55,4 g (89\% d. Th.) kristallines Hydrazon (2) vom Schmp. $198-202{ }^{\circ} \mathrm{C}$ (aus Methanol/Wasser). Auf eine weitere Reinigung wurde verzichtet und das rohe Hydrazon zur weiteren Reaktion eingesetzt.

\section{2. $3 \beta$-Hydroxy-5a-chlor-17-jod-6 $\beta, 19-\Delta^{16}$-androsten}

53,6 g $3 \beta$-Hydroxy- $5 \alpha$-chlor- $6 \beta$, 19-epoxy-androstan17-on-hydrazon (2) wurden in $1,5 \mathrm{l}$ peroxidfreiem Tetrahydrofuran gelöst und $270 \mathrm{ml}$ Triäthylamin zugefügt. Unter Rühren und Eiskühlung wurden $76,6 \mathrm{~g}$ Jod in kleinen Portionen zugegeben. Bei jeder Jodzugabe war eine lebhafte Gasentwicklung festzustellen. Es wurde darauf geachtet, daß die Reaktionstemperatur $25{ }^{\circ} \mathrm{C}$ nicht überschritt. Allmählich schied sich während der Jodzugabe auch Triäthylaminhydrojodid aus. Die Jodzugabe wurde beendet, als eine Braunfärbung be-

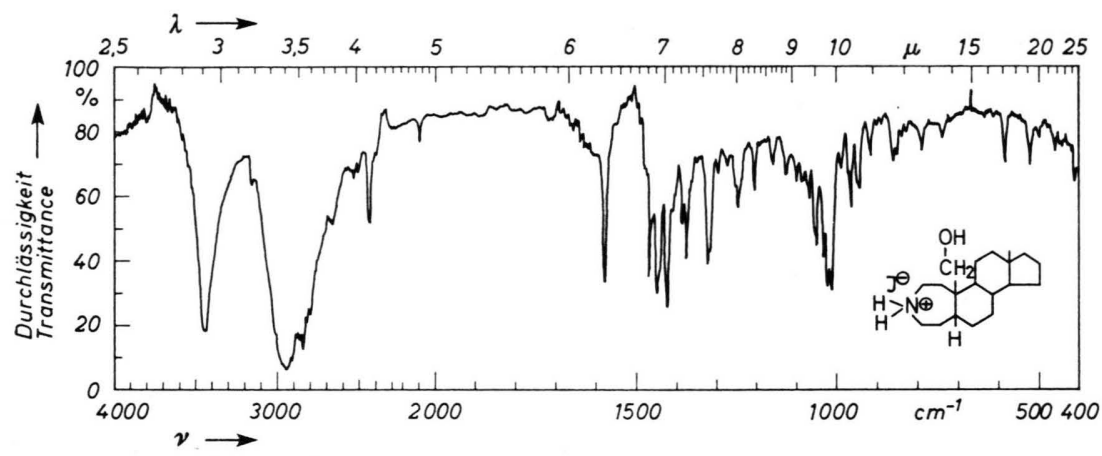

Abb. 3. IR-Spektrum des 19-Hydroxy-3-aza-A-homo- $5 \beta$-androstan- $\mathrm{HJ}$ in $\mathrm{KBr}$.

Abb. 4. IR-Spektrum des 19-Oxo3 -aza-A-homo- $5 \beta$-androstan $\mathrm{HJ}$ in

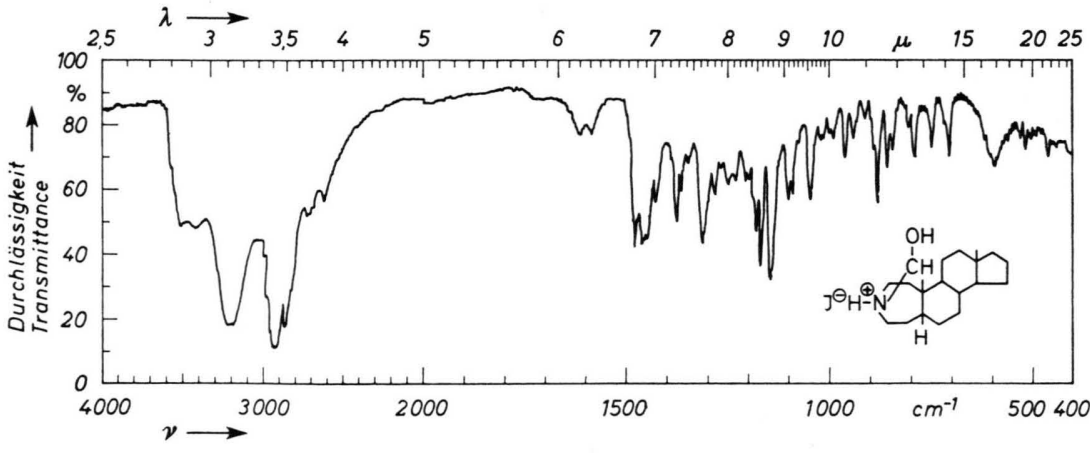
$\mathrm{KBr}$.

8 C. Schöpf u. O. Müller, Liebigs Ann. Chem. 633, 127 [1960]. 
stehen blieb und keine Gasentwicklung mehr auftrat. Nun wurde das Reaktionsgemisch noch 2 Stdn. bei Raumtemperatur stehengelassen und danach mit kalter 2-n. $\mathrm{H}_{2} \mathrm{SO}_{4}$ angesäuert und mit $\mathrm{CH}_{2} \mathrm{Cl}_{2}$ mehrmals extrahiert. Die vereinigten $\mathrm{CH}_{2} \mathrm{Cl}_{2}$-Extrakte wurden erst mit 2-n. $\mathrm{H}_{2} \mathrm{SO}_{4}$, dann mit $\mathrm{Na}_{2} \mathrm{SO}_{3}$-Lösung gewaschen, wobei sich die dunkelbraun gefärbte $\mathrm{CH}_{2} \mathrm{Cl}_{2}$-Lösung bis zur schwachen Gelbfärbung aufhellte. Der $\mathrm{CH}_{2} \mathrm{Cl}_{2}-\mathrm{Ex}$ trakt wurde dann mit gesättigter $\mathrm{NaHCO}_{3}$-Lösung und $\mathrm{H}_{2} \mathrm{O}$ neutral gewaschen und nach dem Trocknen über $\mathrm{Na}_{2} \mathrm{SO}_{4}$ eingedampft. Man erhielt $37,8 \mathrm{~g}$ (57\% d. Th.) kristallines 3 vom Schmp. $152-153{ }^{\circ} \mathrm{C}$ (aus Methanol), auf dessen weitere Reinigung ebenfalls verzichtet wurde und das gleich zur weiteren Reaktion eingesetzt wurde.

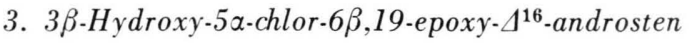

61,5 g $3 \beta$-Hydroxy-5 $\alpha$-chlor-17-jod- $6 \beta, 19$-epoxy- $\Delta^{16}$. androsten (3) wurden in $2,1 l$ abs. Tetrahydrofuran gelöst und $12 \mathrm{~g} \mathrm{LiAlH}_{4}$, suspendiert in $200 \mathrm{ml}$ Tetrahydrofuran, zugetropft. Nach $5^{1 / 2}$-stdg. Kochen am Rückfluß wurde das überschüssige $\mathrm{LiAlH}_{4}$ mit eiskaltem Methanol zersetzt und die Reaktionsmischung mit Methylenchlorid verdünnt. Nun wurde die Reaktionslösung mit 2-n. Schwefelsäure extrahiert und danach mit Wasser, $\mathrm{NaHCO}_{3}$-Lösung und Wasser gewaschen. Nach dem Trocknen über $\mathrm{Na}_{2} \mathrm{SO}_{4}$ wurde das Lösungsmittel abgezogen. Es blieb ein schaumiger Rückstand, der nach Anreiben mit Petroläther $\left(40-60^{\circ} \mathrm{C}\right)$ durchkristallisierte; Schmp. $122-125^{\circ}$ C. Man erhielt 44,2 g rohes 4 .

\section{4. $6 \beta, 19-E$ poxy- $\Delta^{4.16}$-androstadien-3-on (6 a)}

20 g $3 \beta$-Hydroxy-5 $\alpha$-chlor- $6 \beta, 19$-epoxy- $\Delta^{16}$-androsten (4) wurden in $2 l$ Aceton gelöst und auf $10{ }^{\circ} \mathrm{C}$ abgekühlt. Dann wurden in einem Schuß $30 \mathrm{ml}$ wäßrige $\mathrm{CrO}_{3}$-Lösung $\left(26,8 \mathrm{~g} \quad \mathrm{CrO}_{3}+23 \mathrm{ml}\right.$ konz. $\mathrm{H}_{2} \mathrm{SO}_{4}$ auf $100 \mathrm{ml}$ mit Wasser aufgefüllt) hinzugegeben und bei $10-15{ }^{\circ} \mathrm{C} 1$ Stde. lang gerührt. Danach wurden $50 \mathrm{ml}$ Isopropanol dazugegeben und die Temperatur weitere $10 \mathrm{Min}$. bei $10-15^{\circ} \mathrm{C}$ gehalten. Nach Verdünnen mit $800 \mathrm{ml}$ Wasser und $800 \mathrm{ml}$ Methylenchlorid wurde die org. Phase abgetrennt und die wäßrige Phase mehrmals mit Methylenchlorid extrahiert. Die vereinigten Extrakte wurden gewaschen und getrocknet und bei Badtemperatur im Vakuumrotationsverdampfer eingedampft. Der kristallisierte Rückstand wurde in $600 \mathrm{ml}$ Methanol gelöst, $35 \mathrm{~g}$ Kaliumacetat zugefügt, $380 \mathrm{ml}$ Methanol bei Normaldruck abdestilliert (Badtemperatur $90{ }^{\circ} \mathrm{C}$ ), danach i. Vak. bis zum Rückstand eingedampft und nach Zugabe von $500 \mathrm{ml}$ Wasser mit Methylenchlorid extrahiert. Die vereinigten Extrakte wurden gewaschen und getrocknet. Nach dem Eindampfen erhielt man $15 \mathrm{~g}$ eines farblosen Kristallisates. Durch PSC an Kieselgel $\mathrm{PF}_{254+366}$ (Merck), Laufmittel Chloroform/Petroläther $\left(40-60{ }^{\circ} \mathrm{C}\right) \quad 7: 3+5 \%$ Aceton, konnten abgetrennt werden: $5,8 \mathrm{~g}$ 17-Jod-6 $\beta, 19$-epoxy$\Delta^{4.16}$-androstadien-3-on $(6 \mathbf{b})$. Nach dreimaligem Umkristallisieren aus Methanol schmolz $\mathbf{6} \mathbf{b}$ konstant bei $205-207^{\circ} \mathrm{C}$.

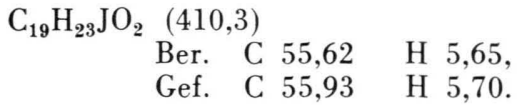

4,7 g $6 \beta, 19-$ Epoxy- $\Delta^{4.16}$-androstadien-3-on (6 a). Nach dreimaligem Umkristallisieren aus Methanol schmolz 6 a konstant bei $123-124{ }^{\circ} \mathrm{C}$.

$$
\begin{array}{rlll}
\mathrm{C}_{19} \mathrm{H}_{24} \mathrm{O}_{2} & (284,4) & & \\
& \text { Ber. } & \text { C } 80,25 & \text { H } 8,50, \\
\text { Gef. } & \text { C } 80,10 & \text { H } 8,56 .
\end{array}
$$

\section{19-Hydroxy- $\Delta^{4.16}$-androstadien-3-on (7 a)}

4,5 g $6 \beta, 19$-Epoxy- $\Delta^{4.16}$-androstadien-3-on (6 a) wurden in $100 \mathrm{ml}$ Eisessig gelöst und mit $35 \mathrm{~g} \mathrm{Zn-Staub}$ versetzt. Der Zinkstaub wurde vorher dreimal mit verd. Essigsäure gewaschen und dann $1^{1 / 2}$ Stdn. lang mit Eisessig aktiviert. Nun wurde $10 \mathrm{Min}$. unter kräftigem Rühren auf $90-100{ }^{\circ} \mathrm{C}$ erwärmt, danach vom überschüssigen Zink abgesaugt, gut mit Eisessig nachgewaschen und das Filtrat im Vakuumrotations-Verdampfer (Badtemperatur $40{ }^{\circ} \mathrm{C}$ ) eingedampft. Der Rückstand wurde mit Chloroform versetzt und nacheinander mit Wasser, $\mathrm{NaHCO}_{3}$-Lösung und Wasser gewaschen. Nach dem Trocknen über $\mathrm{Na}_{2} \mathrm{SO}_{4}$ wurde das Lösungsmittel abgezogen. Man erhielt 3,2 g (71\% d. Th.) eines kristallisierten gelblichen Rückstandes. Durch PSC an Kieselgel $\mathrm{PF}_{254+366}$ (Merck), Laufmittel Chloroform + $5 \%$ Aceton, konnte 7 a weiter aufgereinigt werden. Man erhielt 2,31 g farbloser Kristalle, die nach zweimaligem Umkristallisieren aus Aceton konstant bei $145-147{ }^{\circ} \mathrm{C}$ schmolzen.

$$
\begin{aligned}
& \mathrm{C}_{19} \mathrm{H}_{26} \mathrm{O}_{2}(286,4) \\
& \text { Ber. C 79,68 H 9,15, } \\
& \text { Gef. C } 79,88 \text { H 9,09. }
\end{aligned}
$$

\section{19-Hydroxy-17-jod-4 ${ }^{4.16}$-androstadien-3-on (7 b)}

5 g 17-Jod-6 $\beta, 19$-epoxy- $\Delta^{4.16}$-androstadien-3 on $(\mathbf{6} \mathbf{b})$ wurden in $100 \mathrm{ml}$ Eisessig gelöst und $40 \mathrm{~g}$ Zinkstaub zugegeben, der wie unter 5 aktiviert worden war. Nach 10 Min. kräftigem Rühren und Erwärmen auf 90$100{ }^{\circ} \mathrm{C}$ wurde wie oben aufgearbeitet. Man erhielt $4,21 \mathrm{~g}(82 \%$ d. Th.) rohes kristallines $7 \mathbf{b}$, das durch PSC an Kieselgel $\mathrm{PF}_{254+366}$, Laufmittel Chloroform + $5 \%$ Aceton, weiter gereinigt wurde. Dadurch erhielt man $3,25 \mathrm{~g}$ farblos kristallisierendes $7 \mathbf{b}$, das nach dreimaligem Umkristallisieren aus Aceton konstant bei $188-189{ }^{\circ} \mathrm{C}$ schmolz.

$$
\begin{array}{rrrr}
\mathrm{C}_{19} \mathrm{H}_{25} \mathrm{JO}_{2} & (412,3) & & \\
\text { Ber. } & \text { C } 55,35 & \text { H } 6,11, \\
\text { Gef. } & \text { C } 55,51 & \text { H } 6,16 .
\end{array}
$$

\section{19-Hydroxy-5ß-androstan-3-on (8)}

0,44 g 19-Hydroxy- $\Delta^{4.16}$-androstadien-3-on (7 a) wurden in $15 \mathrm{ml}$ Äthanol gelöst und $0,13 \mathrm{~g} \mathrm{KOH}$ in $15 \mathrm{ml}$ Äthanol zugegeben. Nun wurde in Gegenwart von 0,04 g Pd-C (10-proz., vorreduziert) bei Normaldruck und Raumtemperatur hydriert. Nach Aufnahme von 2 Moläquivalenten Wasserstoff wurde die Hydrierung ab- 
gebrochen. Danach wurde etwas Wasser zugegeben und die vorhandene $\mathrm{KOH}$ mit $2-n . \mathrm{H}_{2} \mathrm{SO}_{4}$ neutralisiert. Zwei Drittel des Lösungsmittels wurden vorsichtig i. Vak. abgezogen und der Rest mit Methylenchlorid verdünnt. Nun wurde die $\mathrm{CH}_{2} \mathrm{Cl}_{2}$-Lösung nacheinander mit 2-n. $\mathrm{H}_{2} \mathrm{SO}_{4}$, Wasser, gesätt. $\mathrm{NaHCO}_{3}$-Lösung und Wasser gewaschen und nach dem Trocknen über $\mathrm{Na}_{2} \mathrm{SO}_{4}$ wurde das Lösungsmittel abdestilliert. Man erhielt 0,435 g (98\% d. Th.) 8, das nach zweimaligem Um. kristallisieren aus Äther konstant bei $109-110^{\circ} \mathrm{C}$ schmolz.

$$
\begin{aligned}
& \mathrm{C}_{19} \mathrm{H}_{30} \mathrm{O}_{2} \quad(290,5) \\
& \text { Ber. C 78,57 H 10,41, } \\
& \text { Gef. C } 78,73 \text { H } 10,20 \text {. }
\end{aligned}
$$

Zum gleichen Ergebnis gelangt man, wenn man 1,95 g 19-Hydroxy-17-jod- $\Delta^{4.16}$-androstadien-3-on (7 b) in $120 \mathrm{ml}$ Äthanol unter Zusatz von $0,8 \mathrm{~g} \mathrm{KOH}$ in $\mathrm{Ge}$ genwart von $0,18 \mathrm{~g}$ vorreduziertem 10 -proz. Pd-C hydriert: 1,32 g 8 (96\% d. Th.).

$$
19 \text { - A c e tox y - } 5 \beta \text { - a nd rostan - } 3 \text { - on (8 a) }
$$

0,43 g 19-Hydroxy-5 $\beta$-androstan-3-on (8) wurden wie üblich mit $4 \mathrm{ml}$ Pyridin und $4 \mathrm{ml}$ Acetanhydrid bei Raumtemperatur acetyliert. Man erhielt 0,43 g (88\% d. Th.) rohes 8 a, das nach zweimaligem Umkristallisieren aus Methanol/Wasser konstant bei $101-102{ }^{\circ} \mathrm{C}$ schmolz.

$$
\begin{aligned}
& \mathrm{C}_{21} \mathrm{H}_{32} \mathrm{O}_{3} \quad(332,5) \\
& \text { Ber. C 75,86 H 9,70, } \\
& \text { Gef. C 75,83 H 9,78. }
\end{aligned}
$$

Aus $0,21 \mathrm{~g} 8$ a wurde das 19 -Acetoxy-5 $\beta$-androstan3-on-oxim (9) wie üblich hergestellt $(25 \mathrm{ml}$ Methanol, $0,67 \mathrm{~g}$ Hydroxylamin- $\mathrm{HCl}+1 \mathrm{~g}$ Natriumacetat in $5 \mathrm{ml}$ Wasser gelöst). Man erhielt $0,21 \mathrm{~g}(95 \%$ d. Th. $) 9$ vom Schmp. $187-188^{\circ} \mathrm{C}$ (Methanol/Wasser).

$$
\begin{aligned}
& \mathrm{C}_{21} \mathrm{H}_{33} \mathrm{O}_{3} \mathrm{~N} \\
& \text { Ber. C 72,59 H 9,57 N 4,03, } \\
& \text { Gef. C 72,67 H 9,41 N 3,97. }
\end{aligned}
$$

\section{19-Acetoxy-3-aza-4-oxo- (10 a) und 19-Acetoxy-4 aza-3-oxo-A-homo-5 $\beta$-androstan

1,5 g 19-Acetoxy-5 $\beta$-androstan-3-on-oxim (9) wurden in $11 \mathrm{ml}$ Thionylchlorid bei $-20{ }^{\circ} \mathrm{C}$ nach B e c k m a n n umgelagert (Versuchsbed. 1. c. ${ }^{7}$ ). Nach der Reaktion wurde die Thionylchloridlösung in $90 \mathrm{ml}$ eiskalte 25-proz. wäßrige $\mathrm{KOH}$ zugetropft und wie üblich $^{7}$ aufgearbeitet. Man erhielt $1,4 \mathrm{~g}$ (93\% d. Th.) rohes kristallisiertes $\varepsilon$-Laktamgemisch vom Rohschmelzpunkt $180-190{ }^{\circ} \mathrm{C}$ (zweimal aus Methanol umkristallisiert). Das $\varepsilon$-Laktam-Gemisch wurde nun durch PSC an $10 \mathrm{Al}_{2} \mathrm{O}_{3}\left(\mathrm{PF}_{254+366}\right.$, Typ T) -Platten getrennt (Laufmittel Benzol/n-Propanol $97: 3$, sechsmalige Entwick-

\footnotetext{
* Die Ausbeute ist deshalb so gering, da aus Reinheitsgrün den nur ein Teil der Zone abgehoben wurde.
}

lung). Man erhielt: $0,625 \mathrm{~g}(42 \%$ d. Th.) $\mathbf{1 0}$ a, das nach Umkristallisieren aus Methanol/Wasser bei 211 $213{ }^{\circ} \mathrm{C}$ schmolz.

$$
\begin{aligned}
& \mathrm{C}_{21} \mathrm{H}_{33} \mathrm{O}_{3} \mathrm{~N} \quad(347,5) \\
& \text { Ber. C 72,59 H 9,57 N 4,03, } \\
& \text { Gef. C 72,64 H 9,47 N 3,93, }
\end{aligned}
$$

und $0,100 \mathrm{~g}\left(7 \%\right.$ d. Th. $\left.{ }^{*}\right) 10 \mathrm{~b}$ vom Schmp. $205-$ $207{ }^{\circ} \mathrm{C}$ (zweimal aus Methanol/Wasser umkristallisiert).

$$
\text { Gef. C } 72,50 \text { H } 9,57 \quad \text { N 3,99. }
$$

\section{19-Hydroxy-3-aza-A-homo-5 $\beta$-androstan}

0,299 g 19-Acetoxy-3-aza-4-oxo-A-homo-5 $\beta$-androstan (10 a) wurden in $50 \mathrm{ml}$ trockenem Tetrahydrofuran mit $0,7 \mathrm{~g} \mathrm{\textrm {LiAlH } _ { 4 }} 20 \mathrm{Stdn}$. am Rückfluß gekocht und wie üblich aufgearbeitet. Man erhielt $0,23 \mathrm{~g}$ (96\% d. Th.) rohes 11, das nach Umkristallisieren aus Äthanol/Wasser bei $82-92{ }^{\circ} \mathrm{C}$ schmolz. Hydrobromid: Schmp. $268^{\circ}$. (Zers.) (Si. ab $\left.262^{\circ} \mathrm{C}\right)$

$$
\begin{aligned}
& \mathrm{C}_{19} \mathrm{H}_{34} \mathrm{BrNO}(372,4) \\
& \text { Ber. C 61,28 H 9,20 N 3,76, } \\
& \text { Gef. C 61,53 H 9,32 N 3,83. }
\end{aligned}
$$

Hydrojodid: Schmp. $248-249{ }^{\circ} \mathrm{C}$ (Zers.; Umkrist. aus Wasser).

$$
\begin{array}{rrrrr}
\mathrm{C}_{19} \mathrm{H}_{34} \mathrm{JNO} & (419,4) \\
\text { Ber. } & \text { C } 54,41 & \text { H } 8,17 & \text { N } 3,34 \text {, } \\
\text { Gef. } & \text { C } 54,45 & \text { H } 8,28 & \text { N } 3,55 .
\end{array}
$$

\section{19-Oxo-3-aza-A-homo-5 $\beta$-androstan (12 b)}

0,09 g 19-Hydroxy-3-aza-A-homo-5 $\beta$-androstan (11) wurden in $20 \mathrm{ml} 3$-proz. Schwefelsäure gelöst, auf dem siedenden Wasserbad erhitzt und sodann $4 \mathrm{ml} 0,2-n$. wäßrige $\mathrm{CrO}_{3}$-Lösung in kleinen Anteilen zugegeben, bis die KJ-Stärke-Reaktion positiv blieb. Danach wurde noch $1 / 4$ Stde. auf dem siedenden Wasserbad erhitzt. Nun wurde die überschüssige $\mathrm{CrO}_{3}$ mit $\mathrm{NaHSO}_{3}$-Lösung reduziert. Beim Abkühlen kristallisierte das Hydrogensulfat von 12 b in Form von Plättchen aus. Es wurde mit 2-n. $\mathrm{NaOH}$ alkalisch gemacht, ausgeäthert und der Äther nach Waschen und Trocknen abgezogen. Man erhielt $0,09 \mathrm{~g}(100 \% \mathrm{~d}$. Th.) eines farblosen Öles, das sofort in das Hydrojodid übergeführt wurde. Aus Wasser umkristallisiert, schmolz das Hydrojodid von 12 b bei $231{ }^{\circ} \mathrm{C}$ (Zers.).

$$
\begin{array}{rrrrr}
\mathrm{C}_{19} \mathrm{H}_{32} \mathrm{JNO} & (417,4) \\
\text { Ber. } & \text { C } 54,68 & \text { H } & 7,73 & \text { N } 3,36, \\
\text { Gef. } & \text { C } 54,66 & \text { H } & 8,02 & \text { N } 3,36 .
\end{array}
$$

Der Deutschen Forschungsgemeinschaft danken wir für die Unterstützung dieser Arbeit, Fräulein GUDRUN MANKe für experimentelle Mithilfe. Herrn Dr. G. SNATZKe, Organisch-Chemisches Institut der Universität Bonn, sind wir für die Aufnahme der CD-Kurven sehr zu Dank verpflichtet. 